EPJ Web of Conferences 66, 06021 (2014)

DOI: $10.1051 /$ epjconf/ 20146606021

(C) Owned by the authors, published by EDP Sciences, 2014

\title{
Polarized Drell-Yan studies at COMPASS
}

\author{
Catarina Quintans ${ }^{1, a}$ on behalf of the COMPASS Collaboration \\ ${ }^{1}$ LIP, 1000-149 Lisbon, Portugal
}

\begin{abstract}
The COMPASS experiment at CERN will soon start a new series of measurements using a pion beam and a transversely polarized target. The study of the polarized Drell-Yan process will provide an insight of the transverse momentum dependent parton distribution functions (TMDs), which is complementary to their extraction from semiinclusive deep inelastic scattering (SIDIS), previously measured in COMPASS. The sign change of Sivers and Boer-Mulders TMDs, when accessed from SIDIS or Drell-Yan, is predicted by theory. Its experimental observation is considered an essential test of the TMD approach.

The experimental aspects of the Drell-Yan measurement in COMPASS are discussed. The set-up optimization, driven by the results of several beam tests are presented, as well as the expected event rates and statistical errors of the azimuthal asymmetries.
\end{abstract}

\section{Introduction}

The COMPASS experiment has been taking data at CERN since 2002, using a polarized muon beam impinging in a polarized target. Among the most important results obtained are the azimuthal spin asymmetries in semi-inclusive deep inelastic scattering of a muon off a transversely polarized proton. These azimuthal asymmetries relate to the convolution of a transverse-momentum dependent parton distribution function (TMD PDF) with the parton fragmentation function into a detected hadron. The full description of the nucleon requires 8 such TMDs, at leading twist and if the intrinsic transverse momentum of partons is not neglected.

More than a decade ago, the polarized Drell-Yan process was pointed out as an alternative way of accessing the TMD PDFs. In this case, the azimuthal spin asymmetries relate to a convolution of 2 TMDs, for the intervening target and beam quark and anti-quark. But while in Drell-Yan an azimuthal spin asymmetry related to the Sivers TMD (unpolarized quark inside a transversely polarized proton) can only arise from initial state interactions, in SIDIS it relates to final state interactions, which results in a sign change of the TMD from one process to the other. This TMD is thus referred to as naive time-reversal odd, just as the Boer-Mulders one. The experimental observation of this sign change is considered a crucial test of the TMD approach.

The azimuthal spin asymmetries measured from the Drell-Yan process relate to 4 modulations in terms of the angle $\phi_{S}$ of the transverse spin vector with respect to the transverse momentum of the virtual photon, measured in the target rest frame; and the angle $\phi$ of one lepton in the Collins-Soper rest frame of the dimuon.

\footnotetext{
a e-mail: Catarina.Quintans@cern.ch
} 
COMPASS will do the first polarized Drell-Yan experiment, starting in 2015. Not only the Sivers and Boer-Mulders sign check can be performed, but more generally the dependence of the TMDs with $x_{F}$ and $p_{T}$ can be studied, as was discussed in the already approved COMPASS proposal [1]. Topics like the $\mathrm{J} / \psi$ polarization, its production mechanisms, and the $\mathrm{J} / \psi$-DY duality, which are closely related, will also be addressed. Despite the fact that the TMDs will be accessed for both SIDIS and DY with the same polarized target and basically the same spectrometer, which is undoubtedly an advantage, the phase space of the two measurements has a limited overlap region. The different average $Q^{2}$ of the 2 measurements has to be taken into account, and the appropriate TMD evolution done.

\section{Experimental set-up}

COMPASS is a fixed target experiment installed in the north area of CERN, using a secondary beam produced from the SPS proton beam. The 2-stage spectrometer allows to detect particles with a wide momentum range, and with large geometrical acceptance, up to $\pm 160 \mathrm{mrad}$. The detailed description of the COMPASS experiment is given at [2]. The Drell-Yan experiment uses a negative pion beam with $190 \mathrm{GeV} / \mathrm{c}$ and a target of ammonia which is polarized transversely to the beam direction. This target material was used in past SIDIS measurements in COMPASS. The protons polarization in the target is $\approx 90 \%$, but a dilution factor of $22 \%$, resulting from the fact that only 3 out of the 17 nucleons in the ammonia molecule are polarizable, must also be taken into account. The target consists of 2 long cells, $55 \mathrm{~cm}$ each, spaced by $20 \mathrm{~cm}$, amounting to 0.6 pion interaction lengths. They are oppositely polarized, and the spin orientation is reversed periodically, in order to cancel some systematic errors. The pairs of muons originated in the Drell-Yan process are detected along the $50 \mathrm{~m}$ of the spectrometer. In order to control the combinatorial background originated from pion and kaon decays into muons, a hadron absorber is placed immediately downstream the polarized target. $\mathrm{Al}_{2} \mathrm{O}_{3}$ is chosen as the suitable material for the $240 \mathrm{~cm}$ long absorber, as it minimizes the muons multiple scattering while maximizing the hadrons stopping power. Inside the absorber and along the beam trajectory, a $120 \mathrm{~cm}$ tungsten beam plug is used to stop the beam which did not interact in the ammonia target.

Figure 1 shows part of the spectrometer, including the target region.

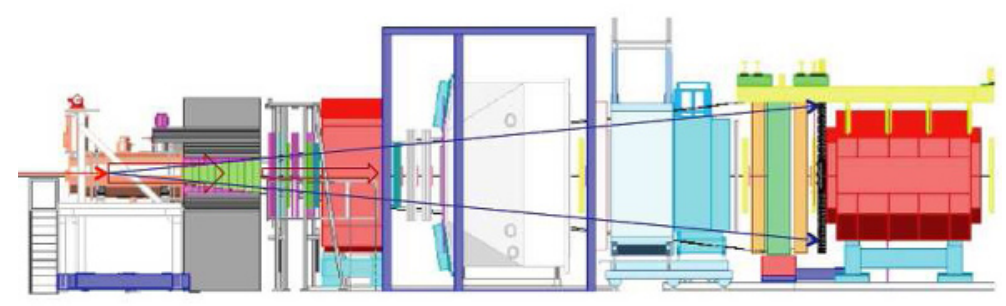

Figure 1. Part of the COMPASS Drell-Yan set-up, including the target. The beam enters from the left-hand side.

Tracker planes placed after thick iron or concrete walls provide the muon identification. The dimuon trigger is made from coincidences of fast hodoscope signals. The hodoscopes are made of horizontal scintillator slabs, and a coincidence matrix of slabs in hodoscope pairs provides some target pointing capability.

The multiple scattering introduced by the hadron absorber limits the vertex reconstruction resolution. In order to improve the situation, a scintillating fiber detector is placed in the middle of the absorber. As a result, the estimated contamination of events reconstructed in the wrong cell is below $1 \%$. 


\section{Acceptance, resolutions and event rates expected}

At COMPASS center-of-mass energy and with $\pi^{-}$beam, the measured Drell-Yan process is dominated by $u-\bar{u}$ quarks annihilation. The signal of dimuon Drell-Yan events competes with several background sources, like the combinatorial of uncorrelated muon pairs, and the physics background of semileptonic decays of open-charm mesons. Also the charmonia resonances contribution should be studied separately. The mass region $4<M_{\mu \mu}<9 \mathrm{GeV} / \mathrm{c}^{2}$ is the most suitable to study Drell-Yan, since there the referred backgrounds can be safely neglected. In COMPASS the Drell-Yan process in this mass region corresponds to the valence region of both target and beam quarks.

The simulation of Drell-Yan events was done using the Pythia generator. From a full Monte Carlo simulation of the spectrometer based in Geant 3 we conclude that the geometrical acceptance for dimuons with mass $4<M_{\mu \mu}<9 \mathrm{GeV} / \mathrm{c}^{2}$ is $39 \%$. The acceptance does not depend on the dimuon $p_{T}$, and it slowly decreases with dimuon mass.

Due to the low cross-section of the Drell-Yan process, in order to maximize the statistics collected one needs the highest beam intensity possible, but several limiting factors must be taken into account. Radiation safety limits have to be respected inside the experimental hall. The stability of the target polarization itself may be disturbed by local heating if the beam intensity is too high. And finally the requirement to have a $190 \mathrm{GeV} / \mathrm{c}$ pion beam with momentum spread below $3 \%$ imposes also an intensity limitation. For these reasons, the pion beam shall not exceed $10^{8}$ particles/second. With a beam intensity of $6 \times 10^{7}$ particles/second, a luminosity of $1.2 \times 10^{32} \mathrm{~cm}^{-2} \mathrm{~s}^{-1}$ can be obtained, which corresponds to 900 Drell-Yan events per day in the mass region $4<M_{\mu \mu}<9 \mathrm{GeV} / \mathrm{c}^{2}$, and $\approx 22500$ events per day in the $\mathrm{J} / \psi$ mass region. In the mass region $2<M_{\mu \mu}<2.5 \mathrm{GeV} / \mathrm{c}^{2}$ one expects 4300 Drell-Yan events per day, but in this region the not-negligible contribution from background needs to be considered. After one year of data taking (which typically corresponds to 140 days) the statistical error in the Sivers asymmetry (integrated over the measured phase space) will be at the level of $2 \%$.

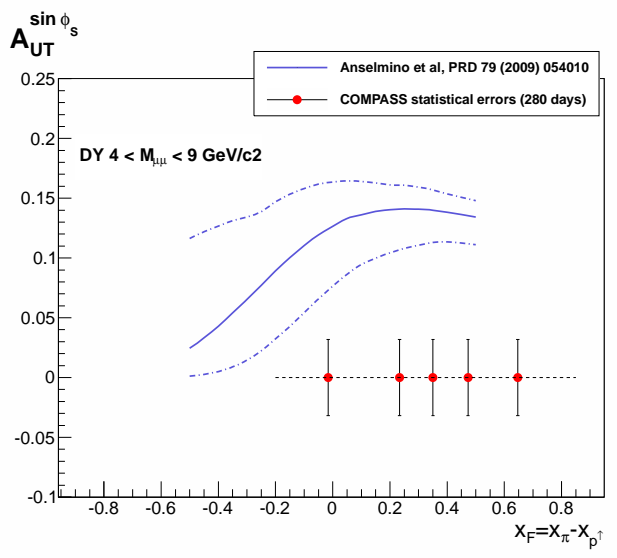

Figure 2. Statistical error on the Sivers asymmetry from Drell-Yan events with $4<M_{\mu \mu}<9 \mathrm{GeV} / \mathrm{c}^{2}$, after 2 years of data-taking. The experimental error bars (in red) are plotted together with one of the theory predictions available (in blue, central value and uncertainty band are shown) [3].

The 4 azimuthal spin asymmetries measured are $A_{U U}^{\cos 2 \phi}, A_{U T}^{\sin \phi_{S}}, A_{U T}^{\sin \left(2 \phi+\phi_{S}\right)}$ and $A_{U T}^{\sin \left(2 \phi-\phi_{S}\right)}$; which relate to the convolution of $\bar{u}$-quark pion Boer-Mulders TMD with $u$-quark proton Boer-Mulders TMD, $\bar{u}$-quark pion unpolarized TMD with $u$-quark proton Sivers TMD, $\bar{u}$-quark pion Boer-Mulders TMD with $u$-quark proton pretzelosity TMD, and $\bar{u}$-quark pion Boer-Mulders TMD with $u$-quark proton transversity TMD, respectively. Table 1 presents their expected statistical errors when integrated over the measured phase-space, for 3 mass ranges of interest, after 2 years of data collection. 
Table 1. Statistical accuracy of the azimuthal spin asymmetries integrated over the experimental kinematic range, after 2 years of data taking. Dimuon mass ranges in units of $\mathrm{GeV} / \mathrm{c}^{2}$

\begin{tabular}{lccc}
\hline uncertainties & $2<M_{\mu \mu}<2.5$ & $2.9<M_{\mu \mu}<3.2$ & $4<M_{\mu \mu}<9$ \\
\hline$\delta A_{U U}^{\cos 2 \phi}$ & 0.0026 & 0.0014 & 0.056 \\
$\delta A_{U T}^{\sin \phi_{S}}$ & 0.0065 & 0.0036 & 0.0142 \\
$\delta A_{U T}^{\sin \left(2 \phi+\phi_{S}\right)}$ & 0.0131 & 0.0073 & 0.0284 \\
$\delta A_{U T}^{\sin \left(2 \phi-\phi_{S}\right)}$ & 0.0131 & 0.0073 & 0.0284 \\
\hline
\end{tabular}

\section{Beam tests}

Several beam tests were already performed, showing the feasibility of the proposed experiment. Measurements done in 2007 allowed to conclude that the target temperature will not increase significantly with the incident pion beam at the proposed intensity, meaning a target polarization relaxation time in the order of thousands of hours. In 2008, using a beam intensity which was $1 / 4$ of the proposed nominal intensity, it was shown that the high occupancies of the detectors closer to the target prevent us from running in "open-spectrometer" configuration. The measurement performed in 2009, having a prototype hadron absorber added immediately downstream the polarized target, has proved its effectiveness to clean the spectrometer from hadronic products, keeping the detector occupancies at reasonable levels. The 2009 beam test used a $190 \mathrm{GeV} / \mathrm{c} \pi^{-}$beam on a 2 cells polyethylene target, $40 \mathrm{~cm}$ each and spaced by $20 \mathrm{~cm}$. The data-taking lasted for 3 days, during which 2 beam intensities were tested: $8 \times 10^{6}$ and $1.4 \times 10^{7} \mathrm{~s}^{-1}$. From the analysis of this data it was possible to check that the $\mathrm{J} / \psi$ events observed matched the expected number. Also the mass and vertex resolutions were in agreement with those expected, thus validating our Monte-Carlo chain. Finally, a short beam test performed in 2012 allowed to positively check the dimuon trigger concept, using 2 large area hodoscopes in coincidence.

\section{Summary}

After several successful tests that have proved the feasibility of the proposed Drell-Yan measurement, COMPASS is going to perform the first polarized Drell-Yan measurement in 2015. The goal of observing the Sivers and Boer-Mulders TMDs predicted sign change should be reached after one year of data collection. More detailed studies of the TMDs, including dependencies with $x_{F}$ and dimuon $p_{T}$ are possible with the accumulated statistics of a second year of running, which could happen from 2018 on, depending on the LHC/CERN future schedule. The proposed measurements are expected to have a significant impact in the knowledge of the proton and pion TMDs.

\section{References}

[1] COMPASS Collaboration, CERN-SPSC-2010-014, SPSC-P-340, 17 May 2010

[2] COMPASS Collaboration, P. Abbon et al., Nucl. Instr.and Meth A 577, 455-518 (2007)

[3] M. Anselmino et al., Phys. Rev. D 79, 054010 (2009) 